\section{Neurotrophic keratopathy and diabetes mellitus}

\begin{abstract}
Diabetes mellitus is frequently associated with microvascular complications such as retinopathy, nephropathy, and peripheral neuropathy. Neurotrophic keratopathy occurs in response to a neuropathy of the ophthalmic division of the trigeminal nerve. Rarely has diabetic neurotrophic keratopathy been described. This paper discusses the ophthalmic histories of three patients who presented with diabetic neurotrophic keratopathy. In one patient the corneal ulceration was the sole presenting feature of his diabetes. We discuss the need for increased vigilance in the ophthalmic community for suspecting diabetes in patients with unexplained corneal epithelial disease. Eye (2006) 20, 837-839. doi:10.1038/sj.eye.6702053; published online 7 October 2005
\end{abstract}

Keywords: neurotrophic; keratopathy; diabetes

\section{Introduction}

Neurotrophic keratopathy is a condition that occurs in patients with a neuropathy affecting the ophthalmic division of the trigeminal nerve. Diabetes mellitus is a common disease associated with microvascular complications such peripheral neuropathy. Diabetes has rarely been associated with neurotrophic keratopathy. ${ }^{1}$ We describe three patients with diabetic neurotrophic keratopathy, of which one was previously unknown to have diabetes, in whom corneal ulceration was the sole presenting feature.

\section{Case reports}

Case 1 A 26-year-old woman with a 10-year history of insulin-dependant diabetes developed a corneal problem. She had no symptoms of external eye disease. She had had extensive laser photocoagulation for proliferative diabetic retinopathy, but there was no history of previous corneal trauma or herpes simplex infection. Ocular examination revealed a best-corrected visual acuity of 6/12 in both eyes. There was an epithelial irregularity extending across the inferonasal quadrant of the right cornea. A diagnosis of exposure keratopathy was made. She was treated with artificial tears and a month later the epithelium had healed. A year later, an epithelial defect in the same area was seen at follow-up. Again she was asymptomatic and the visual acuity was unchanged. Decreased corneal sensation was noted using a cotton swab to touch the peripheral and central cornea. A diagnosis of diabetic neurotrophic keratopathy was made. She was treated with ocular lubricants. After 6 months, the defect had increased in size to $5.5 \mathrm{~mm}$. There was associated corneal neovascularisation. Weak topical steroids were added and with the use of occlusive padding at night, the corneal epithelial defect improved. After 2 months, the cornea had healed. After 2 years, the corneal epithelium had remained intact.

Case 2 A 33-year-old woman with a 24-year history of insulin-dependent diabetes was seen by us for quarterly review. She had a past history of treated proliferative retinopathy in both eyes with two vitreous haemorrhages. There was peripheral neuropathy with a neuropathic foot ulcer. She had no ocular symptoms. On examination, she had a corrected visual acuity of $6 / 12$ in both eyes. There was an epithelial defect with scalloped edge in the inferior part of the left cornea. Corneal sensation was markedly reduced, in the left eye more than the right. A diagnosis of diabetic neurotrophic keratopathy was made. She was treated with a temporary ptosis by intramuscular botulinum toxin (15 U), artificial tears, chloramphenicol ointment, and temporary punctual plugs. Over the next 8 months with intensive lubricant treatment, the ulcer gradually healed and within 12 months the only remaining feature was a stromal scar (Figure 1).
${ }^{1}$ Birmingham and Midland Eye Centre, City Hospital, Birmingham, UK

${ }^{2}$ Worcester Royal Infirmary, Charles Hastings Way, Worcester, UK

Correspondence:

M Hope-Ross, Birmingham and Midland Eye Centre, City Hospital, Dudley Road, Birmingham B18 7QH, UK Tel: + 441215543801 ; Fax: + 441213786183 E-mail: monique. hope-ross@goodhope. nhs.uk

Received: 16 December 2004

Accepted: 28 July 2005 Published online: 7 October 2005 


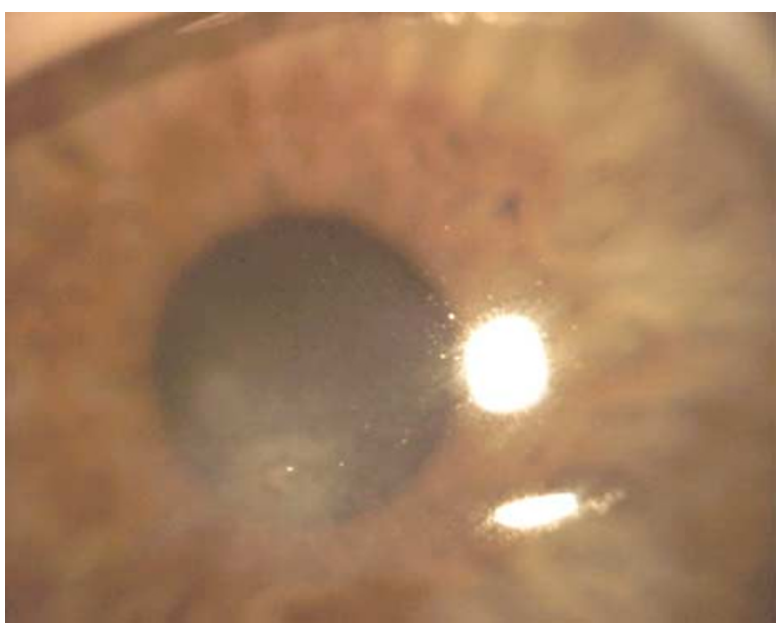

Figure 1 Stromal scar following neurotrophic corneal ulceration (case 2).

Case 3 A 44-year-old man was seen by us for evaluation of corneal ulceration in both eyes. Several weeks before referral, the corneal ulcers were diagnosed. At that time, best-corrected visual acuities were 6/9-3 in the right eye and $6 / 5$ in the left. Despite topical treatment with antibiotics, steroids, and bandage contact lenses, the condition had progressed. There was no previous history of trauma nor was there any ocular or systemic disease. On ocular examination, the best-corrected visual acuities were counting fingers in both eyes. Oval epithelial ulcers extended across the visual axes of the corneas (Figure 2a and $b$ ), and the sensation was greatly reduced. There were high levels of glucose and ketones present on urinalysis, and the blood glucose was $17 \mathrm{mmol} / 1$.

A diagnosis of diabetes mellitus and diabetic neurotrophic keratopathy was made. There was no diabetic retinopathy. Ocular treatment was initially with alternate patching of the eyes together with punctual occlusion and lubricants. Over the course of the year, the corneal epithelial defects healed and visual acuity returned to $6 / 6$ in both eyes.

\section{Comment}

The microvascular complications of diabetes such as retinopathy, nephropathy and peripheral neuropathy are well described. Diabetic retinopathy is the most common cause of visual impairment among persons between 25 and 65 years of age. ${ }^{2}$ Diabetic peripheral neuropathy, a microvascular disease, is characterised by loss of myelinated nerve fibres, wallerian degeneration, and blunted nerve fibre reproduction, the extent of which is associated with the duration of hyperglycaemia. ${ }^{3}$ The mechanism is not well understood; however, the
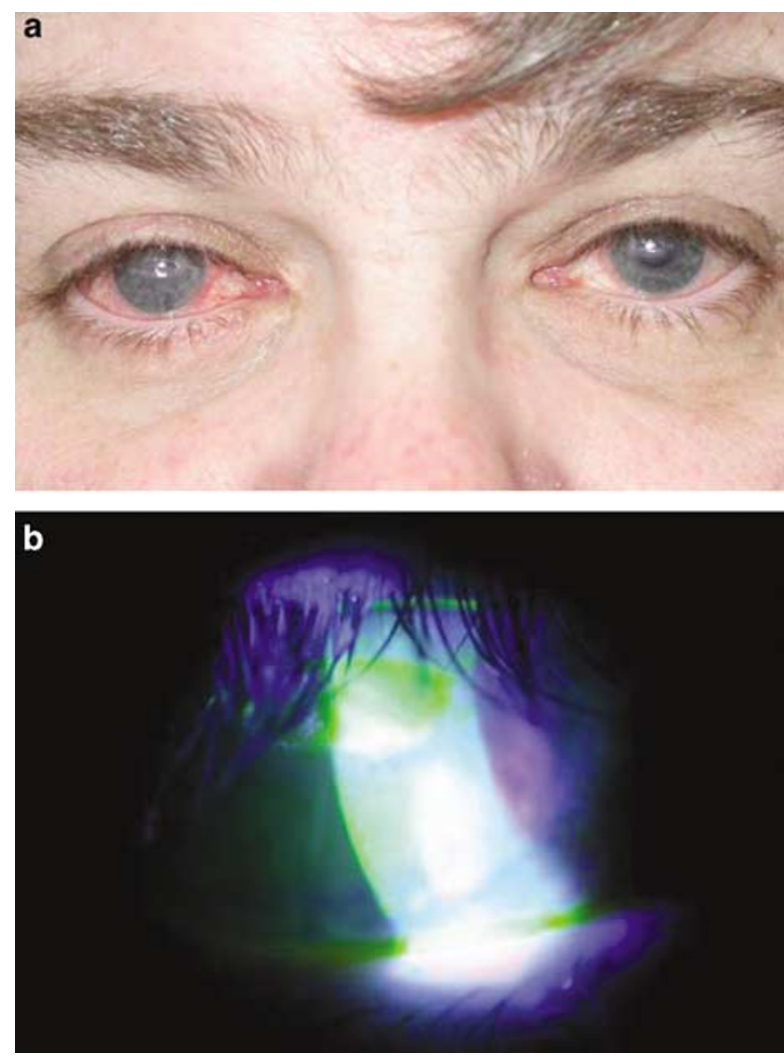

Figure 2 (a) Bilateral corneal ulcers in diabetic neurotrophic keratopathy (case 3). (b) Fluorescein uptake in the corneal epithelial defect of the right eye (case 3).

formation of sorbitol by aldose reductase and advanced glycosylation end products may play a role.

Neurotrophic keratopathy occurs in patients with loss of sensory innervation to the cornea. This is most commonly due to virus infection, such as herpes simplex or herpes zoster, chemical injury, or trauma. ${ }^{4}$

Compression of the trigeminal nerve by aneurysm or tumour may also lead to these corneal changes. Indeed, experimental section of the nerve can lead to neurotrophic ulceration. ${ }^{5}$

Neurotrophic keratopathy progresses in three stages. ${ }^{4}$ The first stage is characterised by epithelial hyperplasia, irregularity, and punctate epithelial erosions. There may be superficial neovascularistion. Stage 2 is characterised by a persistent epithelial defect, around which the edges are smooth and rolled. With stromal involvement (stage 3 ), the ulcer may progress to melting and eventually perforation.

Various neuromediators are postulated to play a role in maintaining epithelial integrity. After sensory denervation, levels of substance $P$ and acetylcholine were depleted in rat corneas. ${ }^{6}$ In vitro, substance $\mathrm{P}$ and acetylcholine induce epithelial proliferation. ${ }^{7}$ Nerve 
growth factor may induce production by sensory nerves of substance $\mathrm{P}$ and acetylcholine. ${ }^{8}$ In addition, nerve growth factor restores corneal integrity and sensation in patients with neurotrophic ulceration. ${ }^{9}$

While rarely described, patients known to have diabetes mellitus can develop neurotrophic keratopathy. ${ }^{1}$ Like neurotrophic keratopathy due to other causes, these patients have decreased corneal sensation and corneal ulcers that fail to respond to antibiotic or steroid therapy. Little is known of the incidence or pathophysiology. Schultz et $a l^{10}$ found reduced corneal sensation in $18 \%$ of a group of randomly selected diabetic patients. A later study found a significant relationship between decreased vibration perception of fingers and toes between diabetic patients with keratopathy and those without, and therefore suggested that the keratopathy was a manifestation of peripheral neuropathy. ${ }^{11}$ Microscopic examination of diabetic corneas shows that the corneal epithelium consists of more enlarged, pleomorphic, and irregularly arranged cells with fewer microvilli, suggestive of impaired ability to heal. ${ }^{12}$

Further evidence that diabetic keratopathy is a manifestation of peripheral neuropathy comes from studies of aldose reductase. Inhibitors of the enzyme improve nerve conduction and regeneration of damaged axons in vitro. ${ }^{13}$ Aldose reductase has been found in most ocular tissues including cornea by immunohistochemistry. ${ }^{14}$ Topical aldose reductase inhibitors in rats prevent the delay in corneal healing seen in rats fed a high-galactose diet. ${ }^{15}$ In humans, aldose reductase inhibitors have been shown to improve sensitivity and morphology. ${ }^{16}$

We now know that the severity of diabetic retinopathy is closely related to blood sugar level and hypertension. ${ }^{2}$ Little is known of the risk factors associated with diabetic neurotrophic keratopathy. One study has suggested a correlation between corneal sensation and severity of retinopathy. ${ }^{17}$ An association between age of patient and corneal sensation in both diabetics and nondiabetics has been shown. ${ }^{18}$ The duration of diabetes in this study was unrelated to corneal sensation.

We describe three patients with diabetes and diabetic neurotrophic keratopathy. In one patient, the corneal disease was the presenting feature of diabetes and to our knowledge this has not been recorded previously in the literature. In the other two patients, corneal disease coexisted with proliferative diabetic retinopathy. Therefore, neurotrophic keratopathy must be considered when a patient with diabetes develops otherwise unexplained corneal epithelial disease, and diabetes mellitus must be considered when a patient presents with an unexplained neurotrophic corneal ulcer.

\section{References}

1 Hyndiuk RA, Kazarian EL, Schultz RO, Seideman S. Neurotrophic corneal ulcers in diabetes mellitus. Arch Ophthalmol 1977; 95: 2193-2196.

2 Infeld DA, O'Shea JG. Diabetic retinopathy. Postgrad Med J 1998; 74: 129-133.

3 Clark CM, Lee DA. Prevention and treatment of the complications of diabetes mellitus. N Engl J Med 1995; 332: 1210-1217.

4 Bonini S, Rama P, Olzi D, Lambiase A. Neurotrophic keratitis. Eye 2003; 17: 989-995.

5 De Haas EBH. Desiccation of cornea and conjunctiva after sensory denervation. Arch Ophthalmol 1962; 67: 79-92.

6 Shimizu T, Izumi K, Fujita S, Koja T, Sorimachi M, Ohba N et al. Capsaicin-induced corneal lesions in mice and the effects of chemical sympathectomy. J Pharmacol Exp Ther 1987; 243: 690-695.

7 Reid TW, Murphy CJ, Iwahashi CK, Foster BA, Mannis MJ. Stimulation of epithelial cell growth by the neuropeptide substance P. J Cell Biochem 1993; 52: 476-485.

8 Levi-Montalcini R. The nerve growth factor 35 years later. Science 1987; 237: 1154-1162.

9 Lambiase A, Rama P, Bomini S, Capriogliio G, Aloe L. Topical treatment with nerve growth factor for corneal neurotrophic ulcers. N Engl J Med 1998; 338: 1174-1180.

10 Schultz RO, Van Horn DL, Peters MA, Klewin KM, Schutten WH. Diabetic keratopathy. Trans Am Ophthalmol Soc 1981; 79: 180-199.

11 Schultz RO, Peters MA, Sobocinski K, Nassif K, Scultz KJ. Diabetic keratopathy as a manifestation of peripheral neuropathy. Am J Ophthalmol 1983; 96: 368-371.

12 Tsubota K, Chiba K, Shimazaki J. Corneal epithelium in diabetic patients. Cornea 1991; 10: 156-160.

13 Yagihashi S, Kamijo M, Ido Y, Mirrlees D. Effects of long-term aldose reductase inhibition on development of experimental diabetic neuropathy. Diabetes 1990; 39: 690-696.

14 Kern TS, Engerman RL. Distribution of aldose reductase in ocular tissues. Exp Eye Res 1981; 33: 175-182.

15 Datiles MB, Kador PF, Fukui HN, Hu TS, Kinoshita JH. Corneal re-epithelialization in galactosemic rats. Invest Ophthalmol Vis Sci 1983; 24: 563-569.

16 Hosotani H, Ohashi I, Yamada M, Tsubota K. Reversal of abnormal corneal epithelial cell morphologic characteristics and reduced corneal sensitivity in diabetic patients by aldose reductase inhibitor, CT-112. Am J Ophthalmol 1995; 119: 288-294.

17 Saito J, Enoki M, Hara M, Morishge N, Chikama T, Nishida T. Correlation of corneal sensation, but not of basal or reflex tear secretion, with the stage of diabetic retinopathy. Cornea 2003; 22(1): 15-18.

18 Murphy PJ, Patel S, Kong N, Ryder REJ, Marshall J. Noninvasive assessment of corneal sensitivity in young and elderly diabetic and nondiabetic subjects. Invest Ophthalmol Vis Sci 2004; 45: 1737-1742. 